\title{
Ripartites tricholoma (Alb. \& Schwein.) P. Karst. (Basidiomycota, Agaricales) un nuevo registro de género y especie para Chile
}

\author{
Ripartites tricholoma (Alb. \& Schwein.) P. Karst. (Basidiomycota, Agaricales) a new record of \\ genus and species for Chile
}

\author{
María José Dibánn ${ }^{1,2,3, *}$, Luis Felipe Hinojosa ${ }^{1,2}$, Viviana Salazar-Vidal(3,4, Götz Palfner \\ ${ }^{1}$ Laboratorio de Paleoecología, Facultad de Ciencias, Universidad de Chile, Las Palmeras 3425, Ñuñoa, RM, Chile. \\ ${ }^{2}$ Instituto de Ecología y Biodiversidad, Las Palmeras N 3425, Ñuñoa, RM, Chile. \\ ${ }^{3}$ ONG Micófilos, Av. Las Torres 851, San Pedro de la Paz, Región del Biobío, Chile. \\ ¿Laboratorio de Química de Productos Naturales, Facultad de Ciencias Forestales, Universidad de Concepción, Víctor Lamas 1290, Concepción, Región del Biobío, Chile. \\ ${ }^{5}$ Laboratorio de Micología y Micorrizas, Facultad de Cs. Naturales y Oceanográficas, Universidad de Concepción, Víctor Lamas 1290, Concepción, Región del Biobío, Chile. \\ *E-mail: mjdiban@gmail.com
}

\section{ABSTRACT}

Ripartites tricholoma collected in the Nature Reserve Altos de Cantillana, near Santiago is described as a first record of this fungal genus and species for Chile. This is a contribution to the knowledge of fungal diversity in Nothofagus macrocarpa dominated forest close to its northern limit of distribution.

\section{INTRODUCCIÓN}

El género Ripartites P. Karst. comprende ocho especies (Kirk 2019) distribuidas principalmente en zonas templadas del hemisferio norte, siendo Europa la zona con el mayor número de registros (Natarajan \& Raman 1982; Horcajada \& Checa 1983; Gómez-Pignataro 1992; Tolgor et al. 2013; Knudsen \& Vesterholt 2018). En el hemisferio sur se pueden encontrar tres especies, $R$. amparae Singer en la zona subtropical de Argentina (Singer \& Digilio 1952), y dos especies en las zonas templadas, R. tricholoma (Alb. \& Schwein.) P. Karst. en la Patagonia Argentina (Niveiro \& Albertó 2013), y R. helomorphus (Fr.) P. Karsten en bosques de Nothofagus Blume de Nueva Zelanda (Horak 1979). Este género se caracteriza por poseer fructificaciones con el píleo convexo, subumbonado o levemente deprimido, seco a subvíscido, no higrófano, con lamelas arqueadas a levemente decurrentes, estípite central, esporada parda (Bas et al, 1995), y esporas globosas a subglobosas con ornamentaciones verrugosas y de color amarillento. En los ejemplares juveniles, la textura del píleo y estípite es finamente vellosa (Moser 1983).

Ripartites tricholoma se distribuye ampliamente en Europa
(Knudsen \& Vesterholt 2018), y en Sudamérica ha sido reportado para Argentina (Singer 1969; Niveiro \& Albertó 2013), en bosques dominados por Nothofagus dombeyi (Mirb.) Oerst. y Austrocedrus chilensis (D.Don) Pic. Serm. \& Bizzarri (Singer 1971).

En este estudio se reporta el primer hallazgo de $R$. tricholoma en Chile, describiendo e ilustrando las características macroscópicas y microscópicas, aportando información sobre su localización y un mapa con su distribución geográfica en el mundo.

Los ejemplares fueron recolectados en la Reserva Altos de Cantillana, Región Metropolitana (3352'58,62"S; $71^{\circ}$ 0 '8,17"O), entre la hojarasca de un bosque de Nothofagus macrocarpa (A.DC.) F.M. Vázquez \& R.A. Rodr. como especie dominante, y acompañado por Schinus montanus (Phil.) Engler, Kageneckia angustifolia D. Don y Azara petiolaris (D. Don) I.M. Johnst. El sotobosque estaba cubierto por abundante hojarasca de N. macrocarpa pero casi sin cobertura vegetal, con algunos ejemplares de Adiantum chilense Kaulf., Berberis chilensis Gill. ex Hook., Haplopappus foliosus DC., Mutisia ilicifolia Cav., Mutisia decurrens Cav. y Perezia poeppigii Less. 
Para el estudio morfológico de los especímenes, en campo se realizaron observaciones in situ, de atributos diagnósticos como color, textura, aroma, tipo de sustrato y de hábitat, además, se tomaron fotografías de los ejemplares. En el laboratorio se procedió a deshidratar el material a $35^{\circ} \mathrm{C}$ en un deshidratador portátil. Posteriormente, se realizaron preparados de las estructuras micromorfológicas, como cortes del himenio, cutícula y estípite, montadas sobre un portaobjeto en $\mathrm{KOH}$ al 5\%, para observar esporas, basidios, cistidios, fíbulas y tipo de pileipellis bajo microscopio. El material fue depositado en el Fungario de la ONG Micófilos (MICOCL).

\section{DESCRIPCIÓN DE LA ESPECIE}

Ripartites tricholoma (Alb. \& Schwein.) P. Karst. Bidr. Känn. Finl. Nat. Folk 32: 477 (1879).

Basiónimo: Agaricus tricholoma Alb. \& Schwein., Conspectus Fungorum in Lusatiae superioris: 188 (1805); Sinónimos homotípicos: Astrosporina tricholoma (Alb. \& Schwein.) J. Schröt., Kryptogamen-Flora von Schlesien 3.1 (33-40): 577 (1889); Flammula tricholoma (Alb. \& Schwein.) P. Kumm., Der Führer in die Pilzkunde: 82 (1871); Gymnopilus tricholoma (Alb. \& Schwein.) Murrill, N. Amer. Flora (1917); Inocybe tricholoma (Alb. \& Schwein.) Kalchbr., Icones selectae Hymenomycetum Hungariae 2: 34, t. 20:3 (1874); Paxillopsis tricholoma (Alb. \& Schwein.) J.E. Lange, Flora Agaricina Danica 5: VI, VI (1940); Paxillopsis tricholoma (Alb. \& Schwein.) J.E. Lange, Flora Agaricina Danica 4: 49 (1939); Paxillus tricholoma (Alb. \& Schwein.) Quél., Enchiridion Fungorum in Europa media et praesertim in Gallia Vigentium: 92 (1886). Sinónimos heterotípicos: Agaricus helomorphus Fr., Epicrisis Systematis Mycologici: 184 (1838); Agaricus strigiceps Fr., Systema Mycologicum 1: 270 (1821); Flammula strigoceps (Fr.) P. Kumm. (1871); Ripartites metrodii Huijsman, Persoonia 1 (3): 337 (1960).

Píleo plano-convexo con el centro levemente deprimido; margen ciliado, fimbriado, levemente incurvado; de color blanquecino, crémeo, 5-7 mm de diámetro. Lamelas crémeas, adnatas a subdecurrentes. Estípite cilíndrico, hirsuto, concoloro con el píleo, $23 \times 4 \mathrm{~mm}$ (Figs. 1A, 1B, 1C, 1D, 1E). Esporas globosas a subglobosas, 5-6 x 4,5-5 $\mu \mathrm{m}$, débilmente amarillentas, inamiloides, verrugosas, apéndice hilar notorio de hasta $1 \mu \mathrm{m}$, ornamentación de hasta 0,8 $\mu \mathrm{m}$ (Figs. 1G, 1L). Basidios claviformes, 20-30 x 5,5-7 $\mu \mathrm{m}$, tetraesporados con esterigmas de 1-5 $\mu \mathrm{m}$ de largo (Figs. 1F, 1I). Cistidios no observados. Pileipellis en forma de cutis con hifas de 5-6 $\mu \mathrm{m}$ de ancho (Figs. 1J, 1M), fíbulas notorias y abundantes (Fig. $1 \mathrm{H})$, terminaciones hifales cilíndricas a levemente claviformes, $70 \times$ 4-7 $\mu \mathrm{m}$ en el píleo, y 100-160 × 5-8 $\mu \mathrm{m}$ en el estípite (Figs. 1K, 1M).

\section{Material estudiado}

CHILE, Región Metropolitana, Prov. Maipo, Reserva Natural Altos de Cantillana. 1550 m.s.n.m. 1-VII-2017, M.J. Dibán (MICOCL-535)

\section{DistRIBUCIÓN GEOGRÁFICA}

Ripartites tricholoma se distribuye ampliamente en el hemisferio norte, con un numero alto de registros en Europa (Knudsen \& Vesterholt, 2018); también existen registros de Japón (Field Museum), Australia (MEL 2381475A), Nueva Zelanda (PDD 43057), Estados Unidos, Canadá, Costa Rica (New York Botanical Garden Herbarium); en Sudamérica ha sido reportada únicamente en Argentina (Singer, 1969; Niveiro \& Albertó, 2013). Se destaca que se encuentra mayormente en hábitats de micro o macroclima templado (Fig. 2).

\section{COMENTARIOS}

El material estudiado coincide con los caracteres morfológicos macroscópicos descriptos por Singer (1969) para el material colectado en Argentina. Con respecto a los caracteres microscópicos, no se observaron queilocistidios, coincidiendo con Knudsen \& Vesterholt (2018) para colecciones europeas, sin embargo, Singer (1969) describe queilocistidios abundantes, versiformes de 13-17 x 3-6 $\mu \mathrm{m}$ en un ejemplar coleccionado en la provincia de Neuquén, Argentina (M3450, BAFC, 22/04/1964, Singer). Esta especie puede ser confundida con $R$. metrodii, sin embargo, esta última posee el píleo glabro y en ocasiones puede presentar pequeños cilios en el margen, además de tener esporas de mayor tamaño, hasta $8 \mu \mathrm{m}$ de largo (Natarajan \& Raman, 1982).

Existen pocos antecedentes acerca de su función ecológica; está considerado como saprobionte sobre hojarasca de diversos árboles, incluyendo Nothofagus (Singer, 1971). El registro de R. tricholoma bajo Nothofagus macrocarpa en Altos de Cantillana es de particular interés ante el escaso conocimiento de la diversidad fúngica en estos bosques cercanos al límite norte de la distribución de Nothofagus en Sudamérica. 

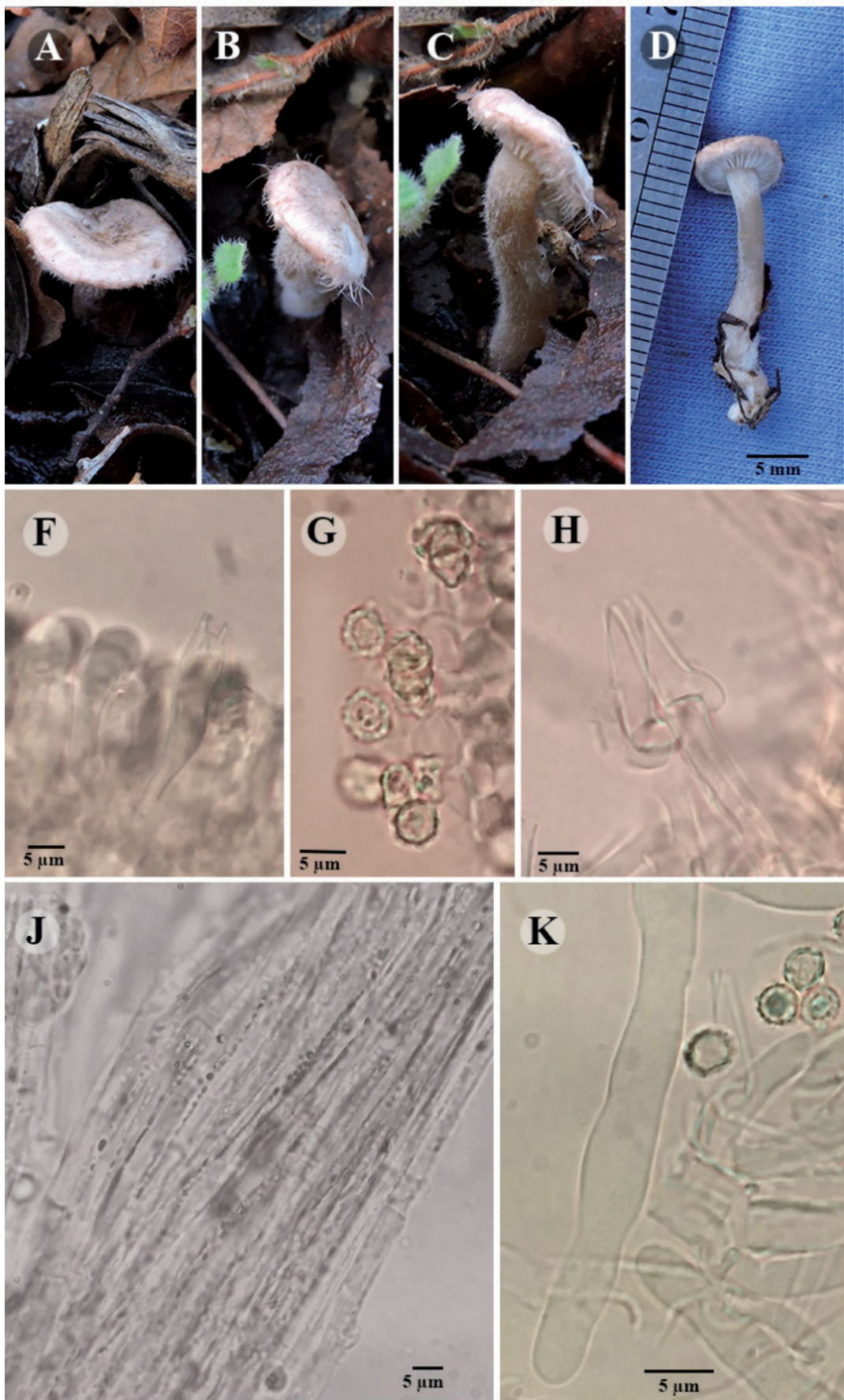
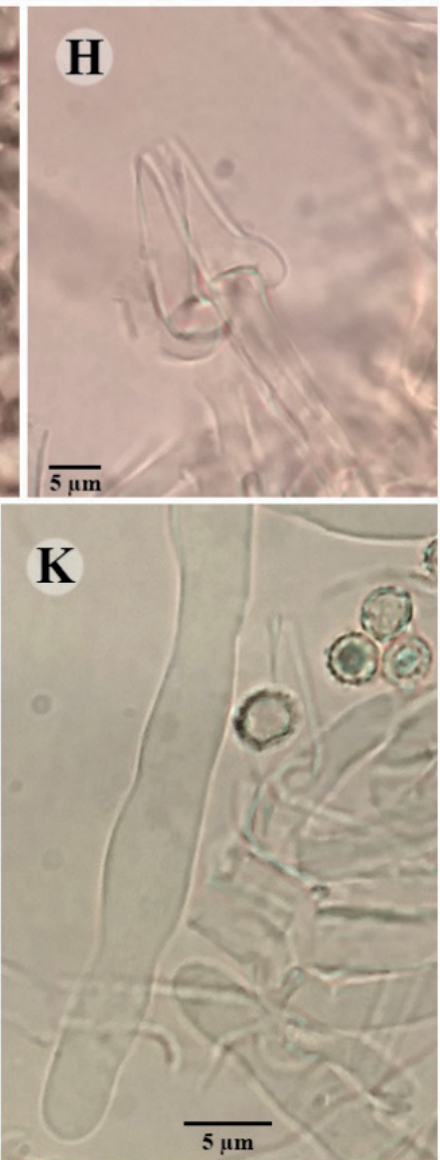

E
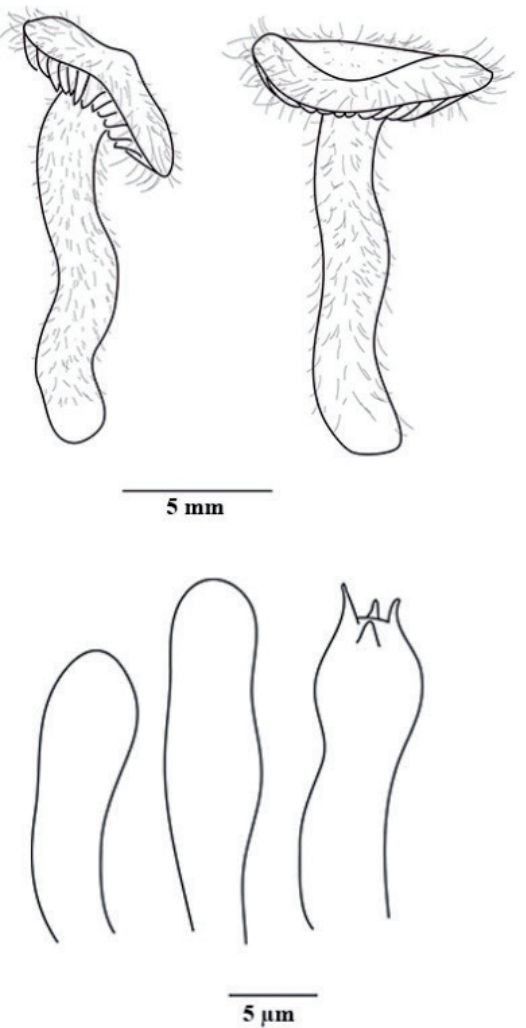

L

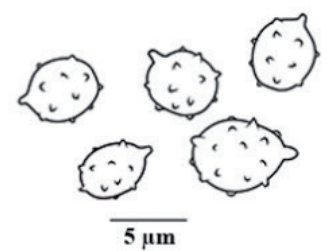

M

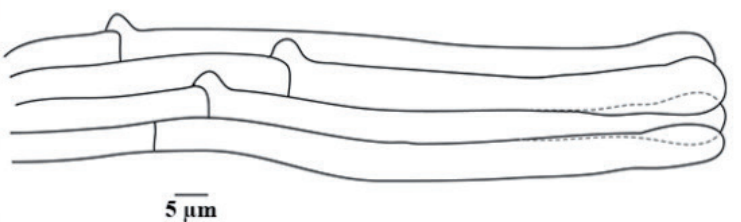

Figura 1. A-C. Basidiomas juveniles de R. tricholoma in situ. D. Referencia de tamaño y detalles del himenio. E. Basidiomas. F-M. Detalles microscópicos a 1000X: F. Basidio y basidiolos. G. Esporas. H. Fíbula. I. Basidio y basidiolos. J. Hifas de las proyecciones ciliadas. K. Terminaciones hifales de la pileipellis. L. Dibujo de las Esporas. M. Pileipellis con sus hifas terminales. / A-C. Fruiting bodies of R. tricholoma in a juvenile state in situ. D. Size reference and details of hymenium. E. Basidiomata. F. Basidium and basidioles. G. Spores. H. Clamp connection. I. Basidium and basidioles. J. Hyphae of ciliate projections. K. Hyphae of pilepellis. L. Spores. M. Pileipellis hyphae. 


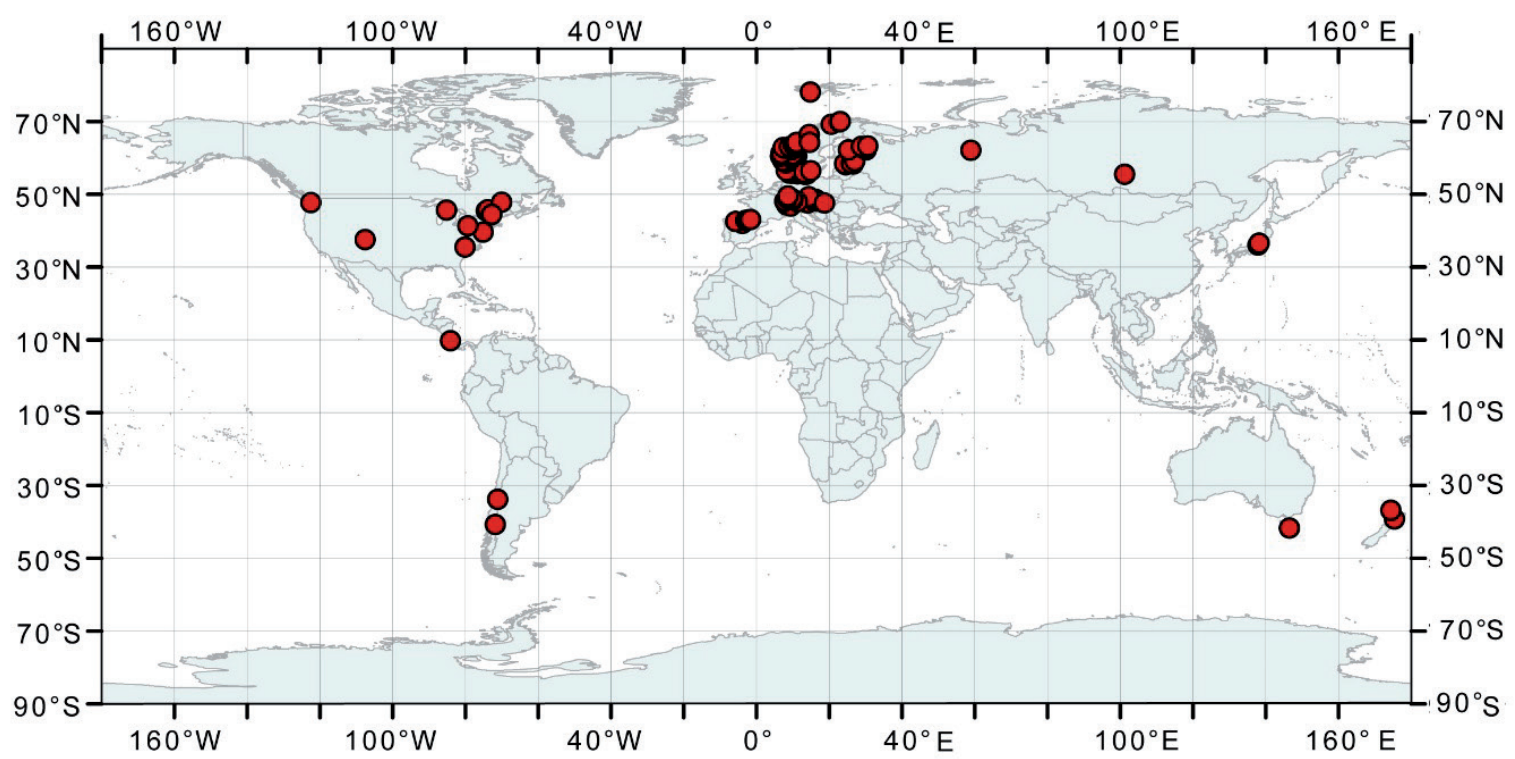

Figura 2. Mapa de distribución de R. tricholoma incluyendo el registro reportado en este artículo. Los círculos rojos indican la posición de colecciones de referencia. Fuente: GBIF. / Distribution map of $R$. tricholoma including the location of this report. Red circles mark locations of reference collections listed in GBIF.

\section{AGRADECIMIENTOS}

A la administración de Reserva Altos de Cantillana y Ximena Romero por los permisos de investigación. A Sebastián Ponce por revisar el artículo. Los autores agradecen a Proyecto Fondecyt 1150690.

\section{REFERENCIAS}

Bas, C., Kuyper, T.W., Noordeloos, M.E., Vellinga, E.C. 1995. Flora Agaricina Neerlandica 3. Tricholomataceae (2). CRCPress, Florida. 200 pp.

Field Museum. 2019. URL: https://www.fieldmuseum.org/. Acceded: Abril 18, 2019.

GBIF. 2019. GBIF Occurrence Download. URL: https://doi. org/10.15468/dl.szdjsi

Gómez, L.D. 1992. Los Basidiomicetes de Costa Rica: V. Paxillaceae (Agaricales, Boletineae). Brenesia 38: 105113.

Horak, E. 1979. Paxilloid Agaricales in Australasia. Sydowia 32(16): 154-166.

Horcajada, G.M., Checa, J. 1983. Estudios sobre Basidiomycetes VIII (Agaricales). Anales del Jardín Botánico de Madrid
40(1): 15-28.

Kirk, P.M. 2019. Species Fungorum (version Oct 2017). In: Species 2000 \& ITIS Catalogue of Life, 20th February 2019. In: Roskov, Y., Ower, G., Orrell, T., Nicolson, D., Bailly, N., Kirk, P.M., Bourgoin, T., DeWalt, R.E., Decock, W., Nieukerken, E., van Zarucchi, J., Penev, L. (Eds.) Species 2000: Naturalis, Leiden, the Netherlands. ISSN 2405-8858. URL: http://www.catalogueoflife.org/col Acceded: May 3, 2019.

Knudsen, H., Vesterholt, J. 2018. Funga Nordica. $2^{\text {nd }}$ Ed. Nordsvamp. Copenhagen, Denmark. 493 pp.

MEL 2381475A, AVH. 2019. The Australasian Virtual Herbarium, Council of Heads of Australasian Herbaria. URL: http:// avh.chah.org.au. Acceded: April 18, 2019.

Moser, M. 1983. Keys to Agarics and Boleti (Polyporales, Boletales, Agaricales, Russulales). Ed. Kibby, G. Transl. Plant, S. London: Roger Phillips. 535 pp.

Natarajan, K., Raman, N. 1982. South Indian Agaricales XIX - A New Species of Ripartites. Mycologia 74(6): 1037-1040.

New York Botanical Garden Herbarium. 2019. URL: http:// sweetgum.nybg.org/science/ Acceded: Abril 18, 2019.

Niveiro, N., Albertó, E.O. 2013. Checklist of the Argentine Agaricales 6. Paxillaceae, Gomphidiaceae, Boletaceae, and Russulaceae. Mycotaxon 123: 429. 
PDD 43057. 2019. Accessed through Systematics Collection Data. URL: http://scd.landcareresearch.co.nz. Acceded: April 18, 2019.

Singer, R. 1969. Mycoflora australis. Beihefte zur Nova Hedwigia 29: 1-405.

Singer, R. 1971. Forest mycology and communities in South America. II. Mycorrhiza sociology and fungus succession in the Nothofagus-Austrocedrus chilensis Woods of
Patagonia. In: Hacskaylor, R. (Ed.) Proceedings of the First North American Conference on Mycorrhizae - USDA Forest Service Publication 1189: 204-215.

Singer, R., Digilio, A.P.L. 1952. Pródromo de la flora agaricina Argentina. Lilloa 25: 5-462.

Tolgor, B., Yu, L., Xin, J. 2013. Two New Records of the Genus Ripartites from China. Journal of Northeast Forestry University (1): 29.

Received: 13.12.2019

Accepted: 10.08.2020 